\title{
INTEGRATION OF HIGHER KORTEWEG-DE VRIES EQUATION WITH SELF-CONSISTENT SOURCE IN CLASS OF PERIODIC FUNCTIONS
}

\author{
M.M. MATYOQUBOV, A.B. YAKHSHIMURATOV
}

\begin{abstract}
In the present work the inverse spectral problem of Sturm-Liouville operator is applied for integrating higher Korteweg-de Vries equation with a self-consistent source in class of periodic functions
\end{abstract}

Keywords: Sturm-Liouville operator, inverse spectral problem, eigenvalue, eigenfunction, Korteweg-de Vries equation.

\section{INTRODUCTION}

In 1967 in work [1] American scientists C.S. Gardner, J.M. Greene, M.D. Kruskal, and R.M. Miura established the integrability of the Korteweg-de Vries equation (KdV) in the class of "fast decaying" w.r.t. $x$ functions by the method of inverse scattering problem for the SturmLiouville equation. In work [2] P. Lax showed an universality of the inverse scattering problem method and generalized the KdV equation by introducing a higher (general) KdV equation.

In works [3-10] KdV equation and higher $\mathrm{KdV}$ equation were studied in the class of finiteband and periodic functions.

In the present work we study the higher $\mathrm{KdV}$ equation with a self-consistent source in the class of periodic functions.

We note that in [11-15] and other papers the KdV equation with a self-consistent source was considered in the class of fast decaying functions, and nonlinear equations with a source in the class of periodic functions in various formulations were studied in works [16-19].

Let

$$
H=-\frac{1}{2} \frac{d^{3}}{d x^{3}}+2 q \frac{d}{d x}+q^{\prime}
$$

where $q=q(x, t)$, and the prime denotes the derivative w.r.t. $x$. According to [20], there exists polynomials $P_{k}$ (of $q$ and the derivatives of $q$ w.r.t. $x$ ) such that

$$
H P_{k}=P^{\prime}{ }_{k+1} \text {. }
$$

For example,

$$
P_{0}=1, \quad P_{1}=q, \quad P_{2}=-\frac{1}{2} q_{x x}+\frac{3}{2} q^{2}, \quad P_{3}=\frac{1}{4} q_{x x x x}-\frac{5}{2} q q_{x x}-\frac{5}{4} q_{x}^{2}+\frac{5}{2} q^{3} .
$$

It is easy to prove the following properties of the operator $H$ (see [20]).

Lemma 1. If $y(x, t)$ is a solution to the following Sturm-Liouville equation

$$
L(t) y \equiv-y^{\prime \prime}+q(x, t) y=\lambda y, \quad x \in R^{1},
$$

the identity

$$
H\left(y^{2}\right)=2 \lambda\left(y^{2}\right)^{\prime}
$$

M.M. Matyoqubov, A.B. Yakhshimuratov, Integration of higher Korteweg-De Vries EQUATion WITH SELF-CONSISTENT SOURCE IN CLASS OF PERIODIC FUNCTIONS.

(C)Matyoqubov M.M., Yakhshimuratov A.B. 2013.

submitted December 30, 2011. 
holds true.

Lemma 2. For any $y(x), z(x) \in C^{3}[0, \pi]$ the identity

$$
\int_{0}^{\pi} H z \cdot y d x=\left.\left(-\frac{1}{2} z^{\prime \prime} y+2 q z y+\frac{1}{2} z^{\prime} y^{\prime}-\frac{1}{2} z y^{\prime \prime}\right)\right|_{0} ^{\pi}-\int_{0}^{\pi} z \cdot H y d x
$$

holds true.

The following equation

$$
q_{t}=H P_{N}[q], \quad x \in R^{1}, \quad t>0,
$$

is called a higher $\mathrm{KdV}$ equation. Employing the properties of the operator $H$, we can rewrite this equation as

$$
q_{t}=P_{N+1}^{\prime}[q], \quad x \in R^{1}, \quad t>0 .
$$

For instance, as $N=0,1,2$ we respectively have

$$
q_{t}=q_{x}, \quad q_{t}=-\frac{1}{2} q_{x x x}+3 q q_{x}, \quad q_{t}=\frac{1}{4} q_{x x x x x}-5 q_{x} q_{x x}-\frac{5}{2} q q_{x x x}+\frac{15}{2} q^{2} q_{x} .
$$

\section{Formulation of PROBlem}

In this work we consider the following higher KdV equation with a self-consistent source

$$
q_{t}=P_{N+1}^{\prime}[q]+2 \int_{0}^{\infty} \beta(\lambda, t) s(\pi, \lambda, t)\left(\psi_{+}(x, \lambda, t) \psi_{-}(x, \lambda, t)\right)_{x} d \lambda, t>0, x \in R^{1},
$$

subject to initial condition

$$
\left.q(x, t)\right|_{t=0}=q_{0}(x),
$$

where $q_{0}(x) \in C^{2 N+1}\left(R^{1}\right)$ is a given real function. It is required to find a real function $q(x, t)$ being $\pi$-periodic w.r.t. $x$,

$$
q(x+\pi, t) \equiv q(x, t), \quad t \geq 0, \quad x \in R^{1},
$$

and satisfying the smoothness conditions

$$
q(x, t) \in C_{x}^{2 N+1}(t>0) \cap C_{t}^{1}(t>0) \cap C(t \geq 0) . .
$$

Here $\beta(\lambda, t) \in C([0, \infty) \times[0, \infty))$ is a given real function having the uniform asymptotics $\beta(\lambda, t)=O\left(\frac{1}{\lambda}\right), \lambda \rightarrow \infty, \psi_{ \pm}(x, \lambda, t)$ are the Floquet solutions (normalized by the condition $\left.\psi_{ \pm}(0, \lambda, t)=1\right)$ to the Sturm-Liouville equation

$$
L(t) y \equiv-y^{\prime \prime}+q(x, t) y=\lambda y, \quad x \in R^{1},
$$

$s(x, \lambda, t)$ is the solution to equation (5) satisfying the initial conditions $s(0, \lambda, t)=0, s^{\prime}(0, \lambda, t)=$ 1.

Remark 1. Let us show the uniform convergence of the integral involved in (1). In order to do it, we employ the identity

$$
s(\pi, \lambda, t) \psi_{+}(\tau, \lambda, t) \psi_{-}(\tau, \lambda, t)=s(\pi, \lambda, t, \tau),
$$

where $s(x, \lambda, t, \tau)$ solves the equation

$$
-y^{\prime \prime}+q(x+\tau, t) y=\lambda y, \quad x \in R^{1},
$$

and obeys the initial conditions $s(0, \lambda, t, \tau)=0, s^{\prime}(0, \lambda, t, \tau)=1$.

The asymptotic formulae

$$
c(x, \lambda, t)=\cos \sqrt{\lambda} x+O\left(\frac{1}{\sqrt{\lambda}}\right), \quad s(x, \lambda, t)=\frac{\sin \sqrt{\lambda} x}{\sqrt{\lambda}}+O\left(\frac{1}{\lambda}\right),
$$


and identities

$$
c^{\prime}(x, \lambda, t)=-\sqrt{\lambda} \sin \sqrt{\lambda} x+O(1), \quad s^{\prime}(x, \lambda, t)=\cos \sqrt{\lambda} x+O\left(\frac{1}{\sqrt{\lambda}}\right), \quad(\lambda \rightarrow \infty)
$$

$$
s(\pi, \lambda, t, \tau)=c(\tau, \lambda, t) s(\pi+\tau, \lambda, t)-s(\tau, \lambda, t) c(\pi+\tau, \lambda, t)
$$

imply the estimates

$$
s(\pi, \lambda, t, \tau)=O\left(\frac{1}{\sqrt{\lambda}}\right), \quad \frac{\partial s(\pi, \lambda, t, \tau)}{\partial \tau}=O\left(\frac{1}{\sqrt{\lambda}}\right), \quad(\lambda \rightarrow \infty) .
$$

These estimates and identity (6) ensures the uniform convergence of the integral involved in equation (1).

The aim of the present work is to provide the procedure of constructing a solution $q(x, t)$, $\psi_{ \pm}(x, \lambda, t)$ to problem (1)-(5) in the framework of the inverse spectral problem for the SturmLiouville operator with a periodic coefficient.

\section{PRELIMINARIES}

In this section, for the completeness of the content, we present some basic information concerning the inverse spectral problem for the Sturm-Liouville operator with a periodic coefficient (see $[21-26])$.

Consider the following Sturm-Liouville operator on the axis

$$
L y \equiv-y^{\prime \prime}+q(x) y=\lambda y, \quad x \in R^{1},
$$

where $q(x)$ is a real continuous $\pi$-periodic function.

By $c(x, \lambda)$ and $s(x, \lambda)$ we denote the solutions to equation (7) satisfying the initial conditions $c(0, \lambda)=1, c^{\prime}(0, \lambda)=0$ and $s(0, \lambda)=0, s^{\prime}(0, \lambda)=1$. The function $\Delta(\lambda)=c(\pi, \lambda)+s^{\prime}(\pi, \lambda)$ is called Lyapunov function or Hill discriminant.

The spectrum of operator (7) is pure continuous and coincides with the set

$$
E=\left\{\lambda \in R^{1}:-2 \leq \Delta(\lambda) \leq 2\right\}=\left[\lambda_{0}, \lambda_{1}\right] \cup\left[\lambda_{2}, \lambda_{3}\right] \cup \ldots \cup\left[\lambda_{2 n}, \lambda_{2 n+1}\right] \cup \ldots
$$

The intervals $\left(-\infty, \lambda_{0}\right),\left(\lambda_{2 n-1}, \lambda_{2 n}\right), n=1,2, \ldots$ are called gaps. Here $\lambda_{0}, \lambda_{4 k-1}, \lambda_{4 k}$ are the eigenvalues of the periodic problem $\left(y(0)=y(\pi), y^{\prime}(0)=y^{\prime}(\pi)\right)$, and $\lambda_{4 k+1}, \lambda_{4 k+2}$ are that of the antiperiodic problem $\left(y(0)=-y(\pi), y^{\prime}(0)=-y^{\prime}(\pi)\right)$ for equation $(7)$.

Let $\xi_{n}, n=1,2, \ldots$, be the roots to the equation $s(\pi, \lambda)=0$. We observe that $\xi_{n}, n=1,2, \ldots$, coincide with the eigenvalues of the Dirichlet problem $(y(0)=y(\pi)=0)$ for equation (7), and moreover, the belongings $\xi_{n} \in\left[\lambda_{2 n-1}, \lambda_{2 n}\right], n=1,2, \ldots$ are fulfilled.

The numbers $\xi_{n}, n=1,2, \ldots$ with the signs $\sigma_{n}=\operatorname{sign}\left\{s^{\prime}\left(\pi, \xi_{n}\right)-c\left(\pi, \xi_{n}\right)\right\}, n=1,2, \ldots$ are called spectral parameters of problem (7). The spectral parameters $\xi_{n}, \sigma_{n}, n=1,2, \ldots$ and the edges $\lambda_{n}, n=0,1,2, \ldots$ of the spectrum are called spectral data of operator (7). Recovering of the coefficient $q(x)$ by the spectral data is called the inverse spectral problem for operator (7).

The spectrum of the Sturm-Liouville operator with the coefficient $q(x+\tau)$ is independent of the real parameter $\tau$, and the spectral parameters depend on $\tau ; \xi_{n}(\tau), \sigma_{n}(\tau), n=1,2, \ldots$ The spectral parameters satisfy the following Dubrovin system of equations

$$
\begin{aligned}
& \frac{d \xi_{n}}{d \tau}=2(-1)^{n-1} \sigma_{n}(\tau) \sqrt{\left(\xi_{n}-\lambda_{2 n-1}\right)\left(\lambda_{2 n}-\xi_{n}\right)} \times \\
& \times \sqrt{\left(\xi_{n}-\lambda_{0}\right) \prod_{\substack{k=1 \\
k \neq n}}^{\infty} \frac{\left(\lambda_{2 k-1}-\xi_{n}\right)\left(\lambda_{2 k}-\xi_{n}\right)}{\left(\xi_{k}-\xi_{n}\right)^{2}}}, \quad n \geq 1 .
\end{aligned}
$$

The Dubrovin system of equations and the following trace formula

$$
q(\tau, t)=\lambda_{0}+\sum_{k=1}^{\infty}\left(\lambda_{2 k-1}+\lambda_{2 k}-2 \xi_{k}(\tau, t)\right)
$$


give the method for solving the inverse spectral problem.

There are also other trace formulae, for instance, the second and third trace formulae read as

$$
\begin{gathered}
q^{2}(\tau)-\frac{1}{2} q_{\tau \tau}(\tau)=\lambda_{0}^{2}+\sum_{k=1}^{\infty}\left(\lambda_{2 k-1}^{2}+\lambda_{2 k}^{2}-2 \xi_{k}^{2}(\tau)\right), \\
\frac{3}{16} q_{\tau \tau \tau \tau}(\tau)-\frac{3}{2} q(\tau) q_{\tau \tau}(\tau)-\frac{15}{16} q_{\tau}^{2}(\tau)+q^{3}(\tau)= \\
=\lambda_{0}^{3}+\sum_{k=1}^{\infty}\left(\lambda_{2 k-1}^{3}+\lambda_{2 k}^{3}-2 \xi_{k}^{3}(\tau)\right) .
\end{gathered}
$$

Employing Dubrovin system of equations and the first trace formula, E. Trubowitz [25] succeeded to prove theorems relating the analyticity of the potential and the decay of the gaps lengths for the periodic potential of the Sturm-Liouville operator $(7)$; if $q(x)$ is a real analytic $\pi$-periodic function, the lengths $\lambda_{2 n}-\lambda_{2 n-1}$ of the gaps exponentially tend to zero, i.e., there exist the constants $a>0, b>0$ such that $\lambda_{2 n}-\lambda_{2 n-1}<a e^{-b n}, n \geq 1$; and viceversa, if $q(x) \in C^{2}\left(R^{1}\right)$ is a real $\pi$-periodic function and the lengths $\lambda_{2 n}-\lambda_{2 n-1}$ of the gaps exponentially tend to zero, then $q(x)$ is an analytic function.

In 1946 G. Borg proved a unique theorem (Borg's inverse theorem) on the period of the potential of the Hill equation (see [27]): the number $\pi / 2$ is a period of the potential $q(x)$ of the Sturm-Liouville equation (7), if and only if all the roots to the equation $\Delta(\lambda)+2=0$ are double, i.e., if and only if all the gaps with odd indices disappear.

In 1977 (see [28]) H. Hochstadt gave a short proof, and in 1984 a generalization of the Borg's theorem (see [29]). Let $q(x) \in C^{1}\left(R^{1}\right)$ be a real $\pi$-periodic function. The number $\pi / n$ is the period of the potential $q(x)$ of Sturm-Liouville equation (7), if and only if all the gaps whose indices are not divisible by $n$ disappear. Here $n \geq 2$ is a natural number.

\section{MAIN THEOREM}

The main result of the present work is the following theorem.

Theorem 1. Let $q(x, t), \psi_{ \pm}(x, \lambda, t)$ be a solution to problem (1)-(5). Then the spectrum of operator (5) is independent of the parameter $t$, and the spectral parameters $\xi_{n}(t), n=1,2, \ldots$ satisfies an analogue of Dubrovin system of equations,

$$
\begin{aligned}
& \dot{\xi}_{n}=2(-1)^{n-1} \sigma_{n}(t)\left\{\sum_{k=0}^{N}\left(2 \xi_{n}\right)^{N-k} \cdot P_{k}[q(0, t)]+\int_{0}^{\infty} \frac{s(\pi, \lambda, t) \beta(\lambda, t)}{\lambda-\xi_{n}} d \lambda\right\} \times \\
& \times \sqrt{\left(\xi_{n}-\lambda_{2 n-1}\right)\left(\lambda_{2 n}-\xi_{n}\right)} \times \sqrt{\left(\xi_{n}-\lambda_{0}\right) \prod_{\substack{k=1 \\
k \neq n}}^{\infty} \frac{\left(\lambda_{2 k-1}-\xi_{n}\right)\left(\lambda_{2 k}-\xi_{n}\right)}{\left(\xi_{k}-\xi_{n}\right)^{2}}}, \quad n \geq 1,
\end{aligned}
$$

where the sign of $\sigma_{n}(t)$ changes to the opposite under each collision of the point $\xi_{n}(t)$ and the edges of the gap $\left[\lambda_{2 n-1}, \lambda_{2 n}\right]$. Moreover, the initial conditions

$$
\left.\xi_{n}(t)\right|_{t=0}=\xi_{n}^{0},\left.\quad \sigma_{n}(t)\right|_{t=0}=\sigma_{n}^{0}, \quad n \geq 1,
$$

hold true, where $\xi_{n}^{0}, \sigma_{n}^{0}, n \geq 1$ are spectral parameters to the Sturm-Liouville operator with the coefficient $q_{0}(x)$.

Proof. We introduce the notation

$$
G(x, t)=2 \int_{0}^{\infty} \beta(\lambda, t) s(\pi, \lambda, t)\left(\psi_{+}(x, \lambda, t) \cdot \psi_{-}(x, \lambda, t)\right)_{x} d \lambda
$$


and rewrite equation (1) as

$$
q_{t}=P_{N+1}^{\prime}[q]+G(x, t) .
$$

By $y_{n}(x, t), n=1,2, \ldots$ we denote orthonormalized eigenfunctions to the Dirichlet problem $(y(0)=0, y(\pi)=0)$ for equation (5) with the $\pi$-periodic potential $q(x, t)$ being a solution to equation (10); these eigenfunctions are associated with the eigenvalues $\xi_{n}(t), n=1,2, \ldots$.

Differentiating the identity $\left(L(t) y_{n}, y_{n}\right)=\xi_{n}$ w.r.t. $t$ and employing the symmetricity of the operator $L(t)$, we have

$$
\begin{gathered}
\dot{\xi}_{n}=\left(L \dot{y}_{n}+q_{t} y_{n}, y_{n}\right)+\left(L y_{n}, \dot{y}_{n}\right)=\left(\dot{y}_{n}, L y_{n}\right)+\left(L y_{n}, \dot{y}_{n}\right)+\left(q_{t} y_{n}, y_{n}\right)= \\
=\xi_{n}\left(\left(y_{n}, y_{n}\right)\right)+\left(q_{t} y_{n}, y_{n}\right)=\int_{0}^{\pi} q_{t}(x, t) y_{n}^{2}(x, t) d x .
\end{gathered}
$$

Here $(\cdot, \cdot)$ is a scalar product in the space $L_{2}(0, \pi)$.

Employing (10) and identity $H P_{k}=P_{k+1}^{\prime}$, we rewrite identity (11) as

$$
\dot{\xi}_{n}=\int_{0}^{\pi} y_{n}^{2}(x, t) H P_{N} d x+\int_{0}^{\pi} y_{n}^{2}(x, t) G(x, t) d x .
$$

Employing Lemmata 1 and 2, we convert the following integral

$$
\begin{gathered}
J_{k}=\int_{0}^{\pi} y_{n}^{2}(x, t) H P_{k} d x=\left.\left(-\frac{1}{2} P_{k}^{\prime \prime} \cdot y_{n}^{2}+2 q P_{k} \cdot y_{n}^{2}+\frac{1}{2} P_{k}^{\prime} \cdot\left(y_{n}^{2}\right)^{\prime}-\frac{1}{2} P_{k} \cdot\left(y_{n}^{2}\right)^{\prime \prime}\right)\right|_{0} ^{\pi}- \\
-\int_{0}^{\pi} P_{k} \cdot H\left(y_{n}^{2}\right) d x=-P_{k}[q(0, t)] \cdot\left[y_{n}^{\prime 2}(\pi, t)-y_{n}^{\prime 2}(0, t)\right]-\int_{0}^{\pi} P_{k} \cdot 2 \xi_{n}\left(y_{n}^{2}\right)^{\prime} d x= \\
=-P_{k}[q(0, t)] \cdot\left[y_{n}^{\prime 2}(\pi, t)-y_{n}^{\prime 2}(0, t)\right]+2 \xi_{n} \int_{0}^{\pi} P_{k}^{\prime} \cdot y_{n}^{2} d x,
\end{gathered}
$$

i.e.,

$$
J_{k}-2 \xi_{n} \cdot J_{k-1}=-P_{k}[q(0, t)] \cdot\left[y_{n}^{\prime 2}(\pi, t)-y_{n}^{\prime 2}(0, t)\right] .
$$

Calculating the following sum

$$
\begin{aligned}
& J_{N}-\left(2 \xi_{n}\right)^{N} \cdot J_{0}=\sum_{k=1}^{N}\left(2 \xi_{n}\right)^{N-k} \cdot\left(J_{k}-2 \xi_{n} \cdot J_{k-1}\right)= \\
& =-\left[y_{n}^{\prime 2}(\pi, t)-y_{n}^{\prime 2}(0, t)\right] \cdot \sum_{k=1}^{N}\left(2 \xi_{n}\right)^{N-k} \cdot P_{k}[q(0, t)]
\end{aligned}
$$

and the integral

$$
J_{0}=\int_{0}^{\pi} y_{n}^{2}(x, t) H P_{0} d x=\int_{0}^{\pi} y_{n}^{2}(x, t) q_{x} d x=-\left[y_{n}^{\prime 2}(\pi, t)-y_{n}^{\prime 2}(0, t)\right],
$$

we deduce the identity

$$
J_{N}=-\left[y_{n}^{\prime 2}(\pi, t)-y_{n}^{\prime 2}(0, t)\right] \cdot \sum_{k=0}^{N}\left(2 \xi_{n}\right)^{N-k} \cdot P_{k}[q(0, t)] .
$$


Now we proceed to calculating the second integral in identity (12),

$$
\int_{0}^{\pi} G \cdot y_{n}^{2} d x=\int_{0}^{\infty} s(\pi, \lambda, t) \beta(\lambda, t)\left\{2 \int_{0}^{\pi} y_{n}^{2} \cdot\left(\psi_{+} \psi_{-}\right)^{\prime} d x\right\} d \lambda .
$$

Integrating by parts, it is easy to see that

$$
\begin{gathered}
I=2 \int_{0}^{\pi} y_{n}^{2} \cdot\left(\psi_{+} \psi_{-}\right)^{\prime} d x=\int_{0}^{\pi}\left\{y_{n}^{2} \cdot\left(\psi_{+} \psi_{-}\right)^{\prime}-\left(y_{n}^{2}\right)^{\prime} \cdot\left(\psi_{+} \psi_{-}\right)\right\} d x= \\
=\int_{0}^{\pi}\left\{y_{n} \psi_{-}\left(y_{n} \psi_{+}^{\prime}-y_{n}^{\prime} \psi_{+}\right)+y_{n} \psi_{+}\left(y_{n} \psi_{-}^{\prime}-y_{n}^{\prime} \psi_{-}\right)\right\} d x .
\end{gathered}
$$

It yields

$$
I=\frac{1}{\xi_{n}-\lambda} \cdot\left[y_{n}^{\prime 2}(\pi, t)-y_{n}^{\prime 2}(0, t)\right]
$$

Hence,

$$
\int_{0}^{\pi} G \cdot y_{n}^{2} d x=\left[y_{n}^{\prime 2}(\pi, t)-y_{n}^{\prime 2}(0, t)\right] \cdot \int_{0}^{\infty} \frac{s(\pi, \lambda, t) \beta(\lambda, t)}{\xi_{n}-\lambda} d \lambda .
$$

Substituting expressions (13) and (14) into (12), we obtain the identity

$$
\begin{gathered}
\dot{\xi}_{n}=\left[y_{n}^{\prime 2}(\pi, t)-y_{n}^{\prime 2}(0, t)\right] \times \\
\times\left\{-\sum_{k=0}^{N}\left(2 \xi_{n}\right)^{N-k} \cdot P_{k}[q(0, t)]+\int_{0}^{\infty} \frac{s(\pi, \lambda, t) \beta(\lambda, t)}{\xi_{n}-\lambda} d \lambda\right\} .
\end{gathered}
$$

Employing the identities

$$
\begin{gathered}
y_{n}(x, t)=\frac{1}{c_{n}(t)} s\left(x, \xi_{n}(t), t\right) \\
c_{n}^{2}(t) \equiv \int_{0}^{\pi} s^{2}\left(x, \xi_{n}(t), t\right) d x=s^{\prime}\left(\pi, \xi_{n}(t), t\right) \frac{\partial s\left(\pi, \xi_{n}(t), t\right)}{\partial \lambda},
\end{gathered}
$$

we have

$$
y_{n}^{\prime 2}(\pi, t)-y_{n}^{\prime 2}(0, t)=\frac{1}{\frac{\partial s\left(\pi, \xi_{n}(t), t\right)}{\partial \lambda}}\left(s^{\prime}\left(\pi, \xi_{n}(t), t\right)-\frac{1}{s^{\prime}\left(\pi, \xi_{n}(t), t\right)}\right) .
$$

Substituting here the expression

$$
s^{\prime}\left(\pi, \xi_{n}, t\right)-\frac{1}{s^{\prime}\left(\pi, \xi_{n}, t\right)}=\sigma_{n}(t) \sqrt{\Delta^{2}\left(\xi_{n}(t)\right)-4}
$$

we obtain

$$
y_{n}^{\prime 2}(\pi, t)-y_{n}^{\prime 2}(0, t)=\frac{\sigma_{n}(t) \sqrt{\Delta^{2}\left(\xi_{n}(t)\right)-4}}{\frac{\partial s\left(\pi, \xi_{n}(t), t\right)}{\partial \lambda}} .
$$

Here $\sigma_{n}(t)=\operatorname{sign}\left\{s^{\prime}\left(\pi, \xi_{n}(t), t\right)-c\left(\pi, \xi_{n}(t), t\right)\right\}$.

The expansions

$$
\begin{gathered}
\Delta^{2}(\lambda)-4=4 \pi^{2}\left(\lambda_{0}-\lambda\right) \prod_{k=1}^{\infty} \frac{\left(\lambda_{2 k-1}-\lambda\right)\left(\lambda_{2 k}-\lambda\right)}{k^{4}} \\
s(\pi, \lambda, t)=\pi \prod_{k=1}^{\infty} \frac{\xi_{k}(t)-\lambda}{k^{2}}
\end{gathered}
$$


imply

$$
\begin{aligned}
& y_{n}^{\prime 2}(\pi, t)- y_{n}^{\prime 2}(0, t)=2(-1)^{n} \sigma_{n}(t) \sqrt{\left(\xi_{n}-\lambda_{2 n-1}\right)\left(\lambda_{2 n}-\xi_{n}\right)} \times \\
& \times \sqrt{\left(\xi_{n}-\lambda_{0}\right) \prod_{\substack{k=1 \\
k \neq n}}^{\infty} \frac{\left(\lambda_{2 k-1}-\xi_{n}\right)\left(\lambda_{2 k}-\xi_{n}\right)}{\left(\xi_{k}-\xi_{n}\right)^{2}}} .
\end{aligned}
$$

By (15) and (16) we get (9).

Let us prove the independence of $t$ for the eigenvalues $\lambda_{n}, n=0,1,2, \ldots$ of the periodic and anti-periodic problems for Sturm-Liouville equation (5). By analogy with formula (15) one can show that

$$
\dot{\lambda}_{n}(t)=\int_{0}^{\pi} G(x, t) v_{n}^{2}(x, t) d x,
$$

where $v_{n}(x, t)$ is a normalized eigenfunction of either periodic or antiperiodic problem for SturmLiouville equation (5). Taking into consideration for structure of the function $G(x, t)$ and proceeding as above, we obtain $\dot{\lambda}_{n}(t)=0$. The proof is complete.

\section{COROllaries OF MAIN THEOREM}

Corollary 1. If instead of $q(x, t)$ we consider $q(x+\tau, t)$, the eigenvalues to periodic and antiperiodic problem are independent of the parameters $\tau$ and $t$, and the eigenvalues $\xi_{n}$ of the Dirichlet problem and the signs $\sigma_{n}$ depend on $\tau$ and $t ; \xi_{n}=\xi_{n}(\tau, t), \sigma_{n}=\sigma_{n}(\tau, t)= \pm 1, n \geq 1$. In this case system (9) casts into the form

$$
\begin{aligned}
& \frac{\partial \xi_{n}}{\partial t}=2(-1)^{n-1} \sigma_{n}(\tau, t)\left\{\sum_{k=0}^{N}\left(2 \xi_{n}\right)^{N-k} \cdot P_{k}[q(\tau, t)]+\int_{0}^{\infty} \frac{s(\pi, \lambda, t, \tau) \beta(\lambda, t)}{\lambda-\xi_{n}} d \lambda\right\} \times \\
& \quad \times \sqrt{\left(\xi_{n}-\lambda_{2 n-1}\right)\left(\lambda_{2 n}-\xi_{n}\right)} \times \sqrt{\left(\xi_{n}-\lambda_{0}\right) \prod_{\substack{k=1 \\
k \neq n}}^{\infty} \frac{\left(\lambda_{2 k-1}-\xi_{n}\right)\left(\lambda_{2 k}-\xi_{n}\right)}{\left(\xi_{k}-\xi_{n}\right)^{2}}}, \quad n \geq 1 .
\end{aligned}
$$

Here

$$
s(\pi, \lambda, t, \tau)=\pi \prod_{k=1}^{\infty} \frac{\xi_{k}(t, \tau)-\lambda}{k^{2}} .
$$

Corollary 2. Consider the case $N=2$. In this case differential equation (1) becomes

$$
q_{t}=\frac{1}{4} q_{x x x x x}-5 q_{x} q_{x x}-\frac{5}{2} q q_{x x x}+\frac{15}{2} q^{2} q_{x}+G(x, t),
$$

and Dubrovin system of differential equations (17) is written as

$$
\begin{aligned}
& \frac{\partial \xi_{n}}{\partial t}=2(-1)^{n-1} \sigma_{n}(\tau, t)\left\{4 \xi_{n}^{2}+2 \xi_{n} q-\frac{1}{2} q_{\tau \tau}+\frac{3}{2} q^{2}+\int_{0}^{\infty} \frac{s(\pi, \lambda, t, \tau) \beta(\lambda, t)}{\lambda-\xi_{n}} d \lambda\right\} \times \\
& \quad \times \sqrt{\left(\xi_{n}-\lambda_{2 n-1}\right)\left(\lambda_{2 n}-\xi_{n}\right)} \times \sqrt{\left(\xi_{n}-\lambda_{0}\right) \prod_{\substack{k=1 \\
k \neq n}}^{\infty} \frac{\left(\lambda_{2 k-1}-\xi_{n}\right)\left(\lambda_{2 k}-\xi_{n}\right)}{\left(\xi_{k}-\xi_{n}\right)^{2}}}, \quad n \geq 1 .
\end{aligned}
$$

Employing the following trace formulae

$$
q(\tau, t)=\lambda_{0}+\sum_{k=1}^{\infty}\left(\lambda_{2 k-1}+\lambda_{2 k}-2 \xi_{k}(\tau, t)\right)
$$




$$
q^{2}(\tau, t)-\frac{1}{2} q_{\tau \tau}(\tau, t)=\lambda_{0}^{2}+\sum_{k=1}^{\infty}\left(\lambda_{2 k-1}^{2}+\lambda_{2 k}^{2}-2 \xi_{k}^{2}(\tau, t)\right)
$$

system (20) can be rewritten in a closed form.

Corollary 3. This theorem provides a method for solving problem (19), (2)-(5).

Indeed, denote by $\lambda_{n}, n=0,1,2, \ldots, \xi_{n}(\tau, t), \sigma_{n}(\tau, t), n=1,2, \ldots$, the spectral data of the problem

$$
-y^{\prime \prime}+q(x+\tau, t) y=\lambda y, \quad x \in R^{1} .
$$

Let us find spectral data $\lambda_{n}, n=0,1,2, \ldots, \xi_{n}^{0}(\tau), \sigma_{n}^{0}(\tau), n=1,2, \ldots$ for the equation

$$
-y^{\prime \prime}+q_{0}(x+\tau) y=\lambda y, \quad x \in R^{1} .
$$

We solve then the Cauchy problem $\left.\xi_{n}(\tau, t)\right|_{t=0}=\xi_{n}^{0}(\tau),\left.\sigma_{n}(\tau, t)\right|_{t=0}=\sigma_{n}^{0}(\tau), n=1,2, \ldots$ for Dubrovin system of equations (20). By trace formula (21) we find the solution to problem (19), (2)-(5). Then it is easy to find the Floquet solutions $\psi_{ \pm}(x, \lambda, t)$.

Remark 2. Let us show that the constructed function $q(\tau, t)$ satisfies equation (19). In order to do it, we employ the following Dubrovin system of equations

$$
\begin{aligned}
& \frac{\partial \xi_{n}}{\partial \tau}=2(-1)^{n-1} \sigma_{n}(\tau, t) \sqrt{\left(\xi_{n}-\lambda_{2 n-1}\right)\left(\lambda_{2 n}-\xi_{n}\right)} \times \\
& \times \sqrt{\left(\xi_{n}-\lambda_{0}\right) \prod_{\substack{k=1 \\
k \neq n}}^{\infty} \frac{\left(\lambda_{2 k-1}-\xi_{n}\right)\left(\lambda_{2 k}-\xi_{n}\right)}{\left(\xi_{k}-\xi_{n}\right)^{2}}}, \quad n=1,2, \ldots,
\end{aligned}
$$

and trace formulae (21), (22), as well as (see [26])

$$
\begin{gathered}
\frac{3}{16} q_{\tau \tau \tau \tau}(\tau, t)-\frac{3}{2} q(\tau, t) q_{\tau \tau}(\tau, t)-\frac{15}{16} q_{\tau}^{2}(\tau, t)+q^{3}(\tau, t)= \\
=\lambda_{0}^{3}+\sum_{k=1}^{\infty}\left(\lambda_{2 k-1}^{3}+\lambda_{2 k}^{3}-2 \xi_{k}^{3}(\tau, t)\right) .
\end{gathered}
$$

Dubrovin system (20) and (23) imply

$$
\frac{\partial \xi_{k}}{\partial t}=\left\{4 \xi_{k}^{2}+2 \xi_{k} q-\frac{1}{2} q_{\tau \tau}+\frac{3}{2} q^{2}+\int_{0}^{\infty} \frac{s(\pi, \lambda, t, \tau) \beta(\lambda, t)}{\lambda-\xi_{k}} d \lambda\right\} \frac{\partial \xi_{k}}{\partial \tau}, \quad k \geq 1 .
$$

First trace formula (21) and (25) yield

$$
\begin{aligned}
q_{t}=-2 \sum_{k=1}^{\infty} \frac{\partial \xi_{k}}{\partial t} & =\left(q_{\tau \tau}-3 q^{2}\right) \cdot \sum_{k=1}^{\infty} \frac{\partial \xi_{k}}{\partial \tau}-4 q \sum_{k=1}^{\infty} \xi_{k} \frac{\partial \xi_{k}}{\partial \tau}-8 \sum_{k=1}^{\infty} \xi_{k}^{2} \frac{\partial \xi_{k}}{\partial \tau}+ \\
& +2 \int_{0}^{\infty} \beta(\lambda, t)\left\{\sum_{k=1}^{\infty} \frac{s(\pi, \lambda, t, \tau)}{\xi_{k}-\lambda} \frac{\partial \xi_{k}}{\partial \tau}\right\} d \lambda .
\end{aligned}
$$

Differentiating trace formulae (21), (22), and (24) w.r.t. $\tau$, we obtain

$$
\begin{gathered}
2 \sum_{k=1}^{\infty} \frac{\partial \xi_{k}}{\partial \tau}=-q_{\tau}, \quad 4 \sum_{k=1}^{\infty} \xi_{k} \frac{\partial \xi_{k}}{\partial \tau}=\frac{1}{2} q_{\tau \tau \tau}-2 q q_{\tau}, \\
-2 \sum_{k=1}^{\infty} \xi_{k}^{2} \frac{\partial \xi_{k}}{\partial \tau}=\frac{1}{16} q_{\tau \tau \tau \tau \tau}-\frac{1}{2} q q_{\tau \tau \tau}-\frac{9}{8} q_{\tau} q_{\tau \tau}+q^{2} q_{\tau} .
\end{gathered}
$$


Employing these identities and expansion (18), by (26) we deduce

$$
q_{t}=\frac{1}{4} q_{\tau \tau \tau \tau \tau}-5 q_{\tau} q_{\tau \tau}-\frac{5}{2} q q_{\tau \tau \tau}+\frac{15}{2} q^{2} q_{\tau}+2 \int_{0}^{\infty} \beta(\lambda, t) \frac{\partial s(\pi, \lambda, t, \tau)}{\partial \tau} d \lambda .
$$

It follows from identity (6) that

$$
\begin{gathered}
q_{t}=\frac{1}{4} q_{\tau \tau \tau \tau \tau}-5 q_{\tau} q_{\tau \tau}-\frac{5}{2} q q_{\tau \tau \tau}+\frac{15}{2} q^{2} q_{\tau}+ \\
+2 \int_{0}^{\infty} \beta(\lambda, t) s(\pi, \lambda, t) \frac{\partial}{\partial \tau}\left(\psi_{+}(\tau, \lambda, t) \cdot \psi_{-}(\tau, \lambda, t)\right) d \lambda .
\end{gathered}
$$

Corollary 4. From the results of work [25] we deduce that if the initial function $q_{0}(x)$ is real and analytic, then the lengths $\lambda_{2 n}-\lambda_{2 n-1}$ of the gaps corresponding to this coefficients decay exponentially. Since the lengths of the gaps corresponding to the coefficients $q(x, t)$ are independent of $t$, the function $q(x, t)$ is analytic w.r.t. $x$.

Corollary 5. The generalized Borg's inverse theorem (see [29]) follows that if $q_{0}(x)$ has the period $\frac{\pi}{n}$, the solution $q(x, t)$ to problem (19), (2)-(5) is $\frac{\pi}{n}$-periodic w.r.t. $x$.

The authors are grateful to prof. A.B. Khasanov (Urgench State University, Uzbekistan) for the formulation of the problem and a discussion of the work.

\section{BIBLIOGRAPHY}

1. C.S. Gardner, J.M. Greene, M.D. Kruskal, R.M. Miura. Method for solving the Korteweg-de Vries equation. // Phys. Rev. Lett. 1967. V. 19, No. 19. P. 1095-1097.

2. P.D. Lax. Integrals of nonlinear equations of evolution and solitary waves. // Comm. Pure and Appl. Math. 1968. V. 21, No. 5. P. 467-490.

3. S.P. Novikov. The periodic problem for the Korteweg-de vries equation I. // Funkts. anal. i prilozh. 1974. V. 8, No. 3. P. 54-66. [ Func. Anal. Appl. 1974. V. 8, No. 3. P. 236-246.]

4. B.A. Dubrovin, S.P. Novikov. Periodic and conditionally periodic analogs of the many-soliton solutions of the Korteweg-de Vries equation. // Zh. Eks. Teor. Fiz. 1974. V. 67, No. 12. P. 2131-2143. [JETP. 1974. V. 40, No. 6. P. 1058-1063.]

5. V.A. Marčenko. The periodic korteweg-de vries problem. // Matem. sb. 1974. V. 95, No. 3. P. 331356. [Math. USSR Sb. 1974. V. 24, No. 3. P. 319-344.]

6. B.A. Dubrovin. Periodic problems for the Korteweg-de Vries equation in the class of finite band potentials. // Funkt. anal. i prilozh. 1975. V. 9, No. 3. P. 41-51. [Funct. anal. appl. 1975. V. 9, No. 3. P. 215-223.]

7. A.R. Its, V.B. Matveev. Schrödinger operators with finite-gap spectrum and $N$-soliton solutions of the Korteweg-de Vries equation. // Teor. matem. fiz. 1975. V. 23, No. 1. P. 51-68. [Theor. math. phys. 1975. V. 23, No. 1. P. 343-355.]

8. P. Lax. Periodic solutions of the KdV equations . // Lecture in Appl. Math. AMS. 1974. V. 15. P. 85-96.

9. P. Lax. Periodic Solutions of the KdV equation. // Comm. Pure and Appl. Math. 1975. V. 28, No. 1. P. 141-188.

10. H.P. McKean, E. Trubowitz. Hill's operator and Hyperelliptic Function Theory in the Presence of infinitely Many Branch Points. // Comm. Pure and Appl. Math. 1976. V. 29, No. 2. P. 143-226.

11. V.K. Mel'nikov. Integration method for Korteweg-de Vries equation with a self-consistent source. Preprint. Dubna. 1988.

12. V.K. Mel'nikov. Integration of the nonlinear Schrödinger equation with a source. // Inverse Problems. 1992. V. 8, No. 1. P. 133-147.

13. J. Leon, A. Latifi. Solution of an initial-boundary value problem for coupled nonlinear waves. // J.Phys. A. 1990. V. 23, No. 8. P. 1385-1403. 
14. G.U. Urasboev, A.B. Khasanov. Integrating the korteweg-de vries equation with a self-consistent source and "steplike" initial data. // Teoret. matem. fiz. 2001. V. 129, No. 1. P. 38-54. [Theor. math. phys. 2001. V. 129, No. 1. P. 1341-1356.]

15. A.B. Khasanov, G.U. Urasboev. Integration of general KdV equation with right-hand side in class of fast decaying function // Uzbek. matem. zhurn. 2003. No. 2. P. 53-59. (in Russian).

16. P.G. Grinevich, I.A. Taimanov. Spectral conservation laws for periodic nonlinear equations of the Melnikov type. // Amer. Math. Soc. Transl. Ser. 2, 2008. V. 224. P. 125-138.

17. A.B. Khasanov, A.B. Yakhshimuratov. The Korteweg-de Vries equation with a self-consistent source in the class of periodic functions. // Teoret. matem. fiz. 2010. V. 164, No. 2. P. 214-221. [Theor. math. phys. 2010. V. 164, No. 2. P. 1008-1015.]

18. A. Yakhshimuratov. The nonlinear Schrödinger equation with a self-consistent source in the slass of periodic functions // Mathematical Physics, Analysis and Geometry. 2011. V. 14, No. 2. P. 153169.

19. A.B. Yakhshimuratov. Integrating the Korteweg-de Vries equation with a special free term in the class of periodic functions. // Ufimskii matem. zhurn. 2011. V. 3, No. 4. P. 144-150. [Ufa Math. J. 2011. V. 3, No. 4. P. 141-147.]

20. B.M. Levitan. Inverse Sturm-Liouville Problems. Nauka, Moscow. 1984. [VNU Science Press, Utrecht, 1987.]

21. E.Ch. Titchmarsh. Eigenfunction expansions associated with second-order differential equations. V. 2. Clarendon Press, Oxford. 1960.

22. W. Magnus, W. Winkler Hill's equation. Interscience Wiley, N.Y. 1966.

23. I.V. Stankevich. A certain inverse spectral analysis problem for Hill's equation. // Dokl. SSSR. 1970. V. 192, No. 1. P. 34-37. [Sov. Math. Dokl. 1970. V. 11. P. 582-586.]

24. V.A. Marčenko. I.V. Ostrovskii. A characterization of the spectrum of Hill's operator // Matem. sborn. 1975. V. 97, No. 4. P. 540-606. [Math. USSR Sb. 1975. V. 26, No. 4. P. 493-554.]

25. E. Trubowitz. The inverse problem for periodic potentials. // Comm. Pure and Appl. Math. 1977. V. 30, No. 3. P. 321-337.

26. B.M. Levitan, I.S. Sargsyan. Sturm-Liouville and Dirac operators. Nauka, Moscow. 1988. [Mathematics and Its Applications (Soviet Series), V. 59. Dordrecht, etc.: Kluwer Academic Publishers. 1990.]

27. G. Borg. Eine Umkehrung der Sturm-Liouvilleschen Eigenwertaufgabe // Acta Math. 1946. V. 78, No. 1. P. 1-96.

28. H. Hochstadt. On a Hill's equation with double eigenvalues. // Proc. Amer. Math. Soc. 1977. V. 65, No. 2. P. 343-374.

29. H. Hochstadt. A generalization of Borg's inverse theorem for Hill's equations // J. Math. Anal. Appl. 1984. V. 102, No. 2. P. 599-605.

Mukhammad Makhsudovich Matyoqubov,

Urgench State University,

Kh. Alimdzhan str., 14,

220100, Urgench, Uzbekistan

E-mail: mmm2210410@mail.ru

Alisher Bekchanovich Yakhshimuratov,

Urgench State University,

Kh. Alimdzhan str., 14,

220100, Urgench, Uzbekistan

E-mail: albaron@mail.ru 